\title{
Estrategia de diferenciación: el caso de las empresas industriales
}

\author{
Carlos Ricardo Chirinos Cuadros* \\ Docente Universidad de Lima. Perú \\ Juan Francisco Rosado Samaniego* \\ Alumno Carrera de Ing. Industrial. Lima, Perú
}

Recibido: 19 de julio del 2016 / Aprobado: 17 de agosto del 2016

RESUMEN: En un mercado globalizado con altas exigencias de satisfacción al consumidor, las empresas industriales han adoptado como estrategia genérica la diferenciación. El éxito de una empresa diferenciada radica en el desarrollo de atributos excepcionales del producto y en el desempeño de procesos con un claro enfoque al servicio. Este artículo busca desplegar la estrategia de diferenciación a lo largo de cuatro actividades de la cadena de valor: el diseño de productos, las compras, la transformación y la distribución.

Palabras clave: diferenciación del producto / ventaja competitiva / cadena de valor / industria

\section{Differentiation: the case of industrial enterprises}

ABstract: In a global market with high demands to satisfy the consumer, industrial companies have chosen differentiation as a generic strategy. The success of a differentiated company lies in the development of exceptional attributes of the product and the performance of processes with a clear focus on service. This article seeks to deploy the differentiation strategy along four activities of the value chain: product design, procurement, processing and transportation.

Keywords: product differentiation / competitive advantage / value chain / industry

* Correos electrónicos: cchirino@ulima.edu.pe, 20121138@aloe.ulima.edu.pe 


\section{ANTECEDENTES}

En los últimos diez años, las exigencias del consumidor en cuanto a precio y calidad han llevado a las empresas a buscar un incremento de productividad a través de la eficiencia y estandarización de sus procesos. Adicionalmente, la invasión de productos importados de bajo precio ha ocasionado un aumento en la rivalidad entre los competidores y una caída en la rentabilidad de varios sectores industriales. Sin embargo, en los mercados actuales existen segmentos con altas exigencias de servicio que se constituyen en una oportunidad.

Una forma de aprovechar esta oportunidad es emplear un factor de diferenciación en el producto y servicio. En este contexto, la diferenciación se ha convertido en un elemento clave para el éxito en las industrias, y mucho más en coyunturas de alta competencia. "La diferenciación es hoy, una fórmula mágica que está detrás del éxito de grandes empresas como Apple, Coca Cola, Zara, Google, Walmart, etc." (Peso, 2013).

\section{ESTRATEGIA DE DIFERENCIACIÓN Y VENTAUA COMPETITIVA}

Toda empresa debe asumir una estrategia general que esté enfocada en la creación de una posición estratégica única y valiosa para la empresa. Esta posición competitiva debe generar disparidad con el resto de empresas del sector. Las empresas tienen tres caminos para lograr una mejor posición frente a la competencia (Porter, 1985).

a) El primer camino es la estrategia genérica de costos, la cual ha sido aplicada en la gran mayoría de empresas nacionales, con el fin de buscar eficiencia en los procesos y generar economías de escala que permitan ofrecer al cliente un producto a menor precio.

b) El segundo camino es la estrategia genérica de enfoque aplicada exclusivamente en un nicho de mercado, dependiendo de las necesidades específicas de este grupo de clientes.

c) El tercer camino, el menos empleado y que se constituye en una oportunidad, es la estrategia genérica de diferenciación, que se caracteriza por ofrecer a los clientes productos con valor superior en cuanto al diseño, funcionalidad y servicio.

Si la estrategia genérica da como resultado un desempeño superior, se dice que la empresa ha desarrollado una ventaja competitiva 
(Hill y Jones, 2009). Es decir, las empresas deben desarrollar una o más ventajas competitivas alineadas al logro de una estrategia genérica. Estas ventajas competitivas ${ }^{1}$ se agrupan en cuatro bloques: eficiencia, calidad, servicio e innovación. Su vinculación con la estrategia genérica se muestra en la figura 1.

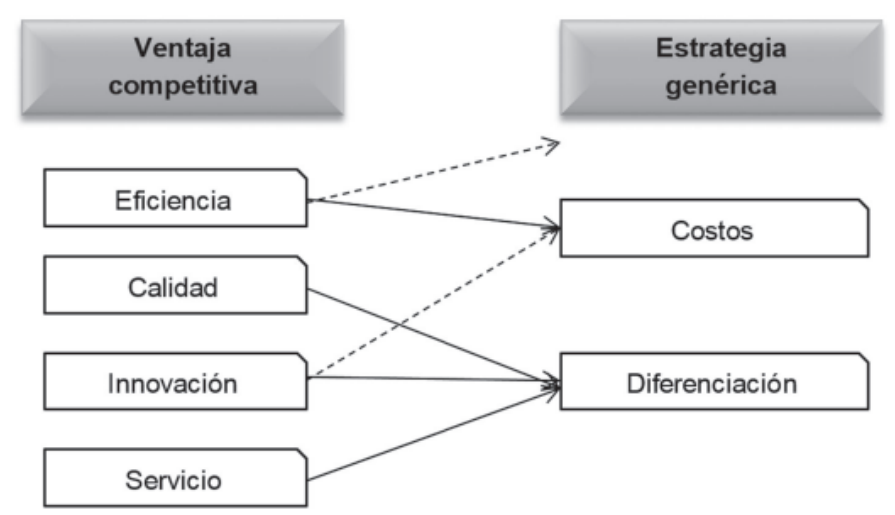

Figura 1. Ventaja competitiva vs. estrategia genérica Elaboración propia

Por ejemplo, para poder implementar la estrategia de diferenciación, la cadena de valor de la empresa debe estar respaldada por la calidad (producto y servicio de altas prestaciones), enfocarse en la innovación (desarrollo e introducción de nuevos productos), sobrepasar las expectativas del cliente y apuntar a un mejor servicio. David (2013) señala que la diferenciación exitosa debe suponer una mayor flexibilidad del proceso, optimizar el diseño de ingeniería, mayor desempeño del producto, mejorar la facilidad de uso, etc.

Es importante anotar que las estrategias de costos y diferenciación no son necesariamente excluyentes. Pueden estar juntas cuando se encuentra el equilibrio correcto entre estas. Por ejemplo, una compañía puede iniciar diferenciándose y con el paso del tiempo generan aumento significativo de la demanda, lo que le permite realizar una economía de escala y, por lo tanto, reducir los costos (Hill, Jones y Schilling, 2015).

1 Una ventaja competitiva significa hacer o tener algo que los rivales deseen (David, 2013) 


\section{EMPRESAS INDUSTRIALES Y DIFERENCIACIÓN EN LA CADENA DE VALOR}

Toda empresa tiene una cadena de valor que abarca el conjunto de actividades que se requieren para llevar a cabo un producto desde la concepción hasta la entrega al consumidor. Para Porter (1985), la cadena de valor es una herramienta que ayuda a comprender las fuentes potenciales de diferenciación. "La estrategia genérica tiene que ser reflejada en una cadena de valor distintiva" (Porter, 2005). La cadena de valor de una empresa industrial está compuesta por seis actividades primarias 2 : planificación, compra, transformación, venta, distribución y postventa, y cuatro actividades de apoyo ${ }^{3}$ : gestión de infraestructura, gestión de recursos humanos, desarrollo tecnológico y adquisición.

La figura 2 muestra las principales actividades de la cadena de valor de una empresa industrial típica.

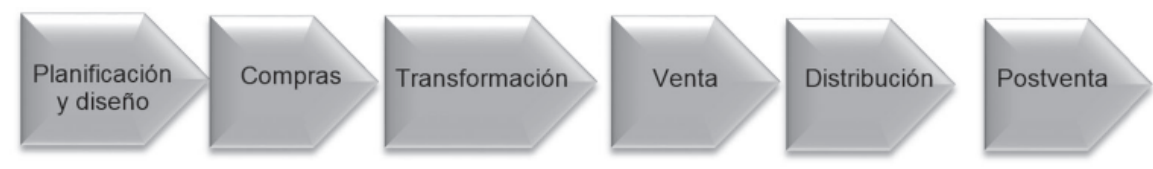

Figura 2. Cadena de valor en una empresa industrial Elaboración propia

A continuación, se propone cómo generar diferenciación a través del desarrollo de ventajas competitivas en las actividades relacionadas a las operaciones de la empresa industrial.

\subsection{DIFERENCIACIÓN EN EL DISEÑO Y DESARROLLO DEL PRODUCTO}

Una forma de diferenciarse de la competencia es ofrecer al mercado un producto que atienda las necesidades de cada cliente, a través de una respuesta rápida y flexible, ofreciendo así una capacidad de personalización casi imposible de igualar por la competencia, en la que las competencias tecnológicas se ligan al saber y a la experiencia acumulada por la empresa (Apocada, Maldonado y Máynez, 2016). El diseño diferenciado

2 Son aquellas que se vinculan directamente con el cliente.

3 Son aquellas que respaldan las actividades primarias. 
debe incluir la definición detallada de las especificaciones del producto como las funciones, la calidad y las partes que lo componen. Es importante que la empresa tenga una organización interna para desarrollar el producto en el menor tiempo posible y con la mayor interrelación con el cliente (Centro de innovación y desarrollo empresarial, 2002).

Para lograr una diferenciación en el diseño y desarrollo de productos, es recomendable aplicar la ingeniería concurrente, que involucra la participación de un equipo multidisciplinario de distintas áreas como la de marketing, ventas, diseño/ingeniería, compras, producción y finanzas. En este proceso, el equipo realiza consultas a agentes externos (clientes y proveedores), para garantizar que el diseño satisface las especificaciones necesarias y que facilitará más adelante la industrialización. Además, se requiere de una innovación tecnológica que añada valor al producto, por ejemplo, herramientas que permitan interactuar con los clientes y proveedores (Centro de Innovación y Desarrollo Empresarial, 2002).

\subsection{DIFERENCIACIÓN EN LAS COMPRAS Y ABASTECIMIENTO}

En el proceso de compras y abastecimiento, una empresa puede lograr diferenciarse si opera cada vez de manera más integrada con el exterior, sobre todo con los proveedores claves, con la finalidad de conseguir una red de colaboración e interrelación con todas aquellas empresas que necesita en su cadena de suministro para lograr un mejor costo, mejor nivel de servicio y garantizar la disposición de materiales de calidad (Shapiro, 2005).

La integración con el proveedor permite visualizarlo como un socio clave, de manera que se le pueda brindar apoyo para la mejora continua de sus procesos y hacerlo partícipe del diseño de los productos que nos ofrece. Para lograr esto, la empresa no debe buscar solamente a proveedores nacionales, sino también a proveedores internacionales y globalizados que estén orientados en la cadena de valor (Centro de Innovación y Desarrollo Empresarial, 2004). La integración con los proveedores no es una decisión aislada; exige cambios en la cadena de valor, sobre todo si se pretende lograr beneficios como la simplificación de los procesos, el aumento de la productividad, un mejor control de los inventarios y una rápida interacción entre los elementos indispensables (Rodriguez, 2003; Muñoz y Zuluaga 2016). 
Otra forma de diferenciarse es a través de la selección correcta del proveedor. Esta debe darse en función de las competencias claves, la flexibilidad ante los cambios, los tiempos de respuesta, la gestión de pedidos y la capacidad de innovación y tecnología del proveedor. Existen, adicionalmente, elementos cualitativos a tener en cuenta, como la fiabilidad del proveedor, su vinculación con la empresa y el hecho de no significar una competencia clave para la empresa o proveer a la competencia de los mismos materiales (Centro de Innovación y Desarrollo Empresarial, 2002, 2004).

\subsection{Diferenciación en la transformación}

Las empresas industriales deben diferenciarse en la transformación, rediseñando los procesos productivos y replanteándose todos los aspectos relacionados con el proceso, empezando con los resultados, la infraestructura, las secuencias, la tecnología, los recursos invertidos y la capacitación de los operarios. El rediseño del proceso productivo tiene que crear un mayor valor en los productos o servicios y logra la optimización de los recursos, menores tiempos, mayor flexibilidad y mejor calidad del producto (Centro de Innovación y Desarrollo Empresarial, 2002).

Para lograr redefinir el proceso de transformación, la empresa tiene que contar con un equipo de trabajo específico que analice los requerimientos de los clientes y del negocio. Se debe entender la situación actual para poder iniciar una etapa de lluvia de ideas y analizar la viabilidad de todas las propuestas de mejora y seleccionar la más rentable para la compañía y el cliente.

Otra forma de diferenciarse es a través de la subcontratación de especialistas, que tengan a cargo los procesos internos que no generen mucho valor al producto o servicio que ofrece la empresa, y lograr así que la compañía concentre sus recursos en diferenciar los procesos internos que sí generan gran valor al producto o servicio.

Contar con la capacidad de flexibilidad en la transformación es otra forma de diferenciarse. El objetivo es mejorar la capacidad de respuesta a las necesidades del cliente, realizar cambios en cantidad o variedad en plena producción, reducir los tiempos de entrega y minimizar los niveles de stock. 
De otro lado, la innovación tecnológica permite tener información de manera más rápida y dar una respuesta veloz. Por este motivo, es vital contar con un sistema informático de control y adquisición de datos industriales. La tecnología de automatización también permite incorporar programas informáticos para que los equipos reaccionen de manera autónoma ante diferentes condiciones del entorno. Todo esto puede generar ventajas que permitan diferenciarse de la competencia. No obstante, si bien contar con estas innovaciones tecnológicas permite obtener un beneficio, para que este sea máximo se debe contar con sistemas organizados, para que las personas no solo puedan gestionar sino también mejorar continuamente (Centro de Innovación y Desarrollo Empresarial, 2004).

\subsection{Diferenciación en la distribución y transporte}

La distribución y el transporte también deben generar diferenciación en las empresas industriales. Para ello, se requiere garantizar un sistema de distribución de productos flexible, rápido y sencillo. Las empresas deben tener procedimientos que les permitan separar y clasificar los productos de la forma más adecuada (cantidad, tipo, código de identificación, etc.), aprovechar las nuevas tecnologías para el seguimiento del transporte, como el GPS, de modo que les permita seguir la ruta y optimizarla, haciendo la entrega más rápida y segura.

Otra forma de diferenciar la distribución es segmentando la red según los productos, ya que no todas las líneas de productos tienen las mismas variables competitivas. En ese sentido, la empresa puede lograr acercar el punto de venta al mercado objetivo o considerar esto como un factor clave para decidir de localización de la planta. Otras organizaciones pueden optar por externalizar el servicio de distribución para aprovechar la flexibilidad y especialización de estas (Centro de Innovación y Desarrollo Empresarial, 2002).

En la figura 3 se presenta una síntesis de las estrategias que una empresa industrial que desea diferenciarse puede implementar para desarrollar ventajas competitivas a lo largo de su cadena de valor. 




Figura 3. Desarrollo de ventajas competitivas en las actividades claves de empresas industriales diferenciadas

Elaboración propia

\section{CONCLUSIONES}

La empresa industrial debe identificar y aplicar estrategias para desarrollar ventajas competitivas en sus actividades. La creación de ventajas competitivas es un proceso permanente, pues aquello que hoy significa algo distintivo puede ser imitado por la competencia y presentar el reto de la búsqueda continua de oportunidades de distinción. Lo único que es permanente es la necesidad de desarrollar ventajas competitivas. 


\section{REFERENCIAS}

Apocada, L., Maldonado, S. y Máynez, A. (2016). La ventaja competitiva, desde la teoría de recursos y capacidades. Revista Internacional Administración \& Finanzas, 9(1), 69-80.

Centro de Innovación y Desarrollo Empresarial. (2002). Guía de gestión de la innovación, Parte I: Diagnóstico. Barcelona: Catalunya Innovación.

Centro de Innovación y Desarrollo Empresarial. (2004). Guías de gestión de la innovación, producción y logística. Barcelona: Catalunya Innovación.

David, F. (2013). Conceptos de administración estratégica. México: Pearson Educación de México

Hill, C. H. y Jones, G. (2009). Administración estratégica. México: Mcgraw-Hill/Interamericana Editores.

Hill, Jones, G. y Schilling, M. (2015). Strategic Management: Theory: An Integrated Approach. Mexico: Cengage Learning Editores.

Jose, P. J. (2013). Diferenciación el ejercicio más difícil. The Marketing Intelligence Review, (28), 5-7. Recuperado dettp://www.daemonquest. com/es/innovacion/pdf_publicaciones/6c8d0b-DQ_MIR_28.pdf

Levitt, T. (1980). Marketing Success through Differentiation of Anything. Harvard Business Review. Recuperado de https:// hbr.org/1980/01/marketing-success-through-differentiation-ofanything/ar/1

Muñoz, R. y Zuluaga, S. (2016). Competitividad y cadenas de abastecimiento en el sector productivo del valle del Cauca. Revista Global De Negocios, 4(1), 77-87.

Porter, M. (1985). Competitive Advantage. Nueva York: First Free Press Edition. Recuperado de http://94.236.206.206/dohodi.net/ books/en/Business\%20Books/Michael\%20Porter/Michael.Porter.-. Competitive.Advantage.pdf

Porter, M. (2011). ¿Qué es la estrategia? Harvard Business Review, 89(11), 100-117. Recuperado de www. hbral.com 
Rodríguez, Y. (2003). ¿Qué es la Cadena de Valor? Boletín Tress. Recuperado de http://www.tress.com.mx/boletin/sept2003/cadena.htm

Shapiro, S. (2005). Innovar para ser Competitivo. Harvard Deusto Marketing \& Ventas, 76-79. Recuperado de http://www.harvarddeusto.com/ 